\title{
THE SECOND LARGEST COMPONENT IN THE SUPERCRITICAL 2D HAMMING GRAPH
}

\author{
REMCO VAN DER HOFSTAD, MALWINA J. LUCZAK, AND JOEL SPENCER
}

\begin{abstract}
The 2-dimensional Hamming graph $H(2, n)$ consists of the $n^{2}$ vertices $(i, j), 1 \leq$ $i, j \leq n$, two vertices being adjacent when they share a common coordinate. We examine random subgraphs of $H(2, n)$ in percolation with edge probability $p$, in such a way that the average degree satisfies $2(n-1) p=1+\varepsilon$. Previous work [7] has shown that in the barely supercritical region $n^{-2 / 3} \ln ^{1 / 3} n \ll \varepsilon \ll 1$, the largest component satisfies a law of large numbers with mean $2 \varepsilon n$. Here we show that the second largest component has, with high probability, size bounded by $2^{8} \varepsilon^{-2} \log \left(n^{2} \varepsilon^{3}\right)$, so that the dominant component has emerged. This result also suggests that a discrete duality principle holds, where, after removing the largest connected component in the supercritical regime, the remaining random subgraphs behave as in the subcritical regime.
\end{abstract}

\section{INTRODUCTION AND MAIN RESULT}

In their seminal work [6], Paul Erdős and Alfred Rényi noted with surprise the development of a giant component in the random graph $G(n, p)$ where each of the $n(n-1) / 2$ possible edges of the complete graph of size $n$ is present with probability $p$ independently of all the other edges. When the average degree $(n-1) p$ satisfies $(n-1) p=1+\varepsilon$, and $\varepsilon$ is positive and fixed (independent of $n$ ), then the largest component will contain a positive proportion of the vertices while the size of the second largest component is only logarithmic in $n$. Today we see this as a phase transition phenomenon exhibiting what mathematical physicists call 'mean-field' behaviour.

For many years, there has been great interest in the barely supercritical phase of $G(n, p)$, that is, the range of $p$ values given by $p=(1+\varepsilon) / n$, where $\varepsilon=\varepsilon(n)$ satisfies $n^{-1 / 3} \ll \varepsilon=o(1)$. For convenience, we can also write $\varepsilon=\lambda n^{-1 / 3}$, where $\lambda=\lambda(n) \rightarrow+\infty$, but does so more slowly than $n^{1 / 3}$. In this phase the dominant component has already appeared. We actually know quite precisely that the largest component, $C_{(1)}$, satisfies $\left|C_{(1)}\right|=2 \varepsilon n\left(1+o_{\mathrm{p}}(1)\right)=2 \lambda n^{2 / 3}\left(1+o_{\mathrm{p}}(1)\right)$ with probability tending to 1 as $n \rightarrow \infty$, where $o_{\mathrm{p}}(1)$ denotes a quantity that converges to zero in probability; and that the second largest component, $C_{(2)}$, satisfies $\left|C_{(2)}\right|=\Theta\left(\varepsilon^{-2} \log \left(n \varepsilon^{3}\right)\right)=$ $\Theta\left(n^{2 / 3} \lambda^{-2} \ln \lambda\right)$ with probability tending to 1 as $n \rightarrow \infty$. Thus, in particular, $\left|C_{(2)}\right| \ll n^{2 / 3} \ll\left|C_{(1)}\right|$. Further, as $\lambda$ increases, the largest component increases in size while the second largest diminishes in size. (Actually, the second largest component is being frequently 'gobbled up' by the dominant component, generally leaving a smaller component as the new second component.) See [1, 10] for the proofs of these results, and [2, 9] for introductions to the field. We feel, speaking quite generally, that an intensive study of the second largest component is vital to enhancing our understanding of percolation phenomena.

We believe that the second largest component should grow until the random structure reaches a critical window. In that critical window, which, for $G(n, p)$, means $p=\left(1+\lambda n^{-1 / 3}\right) / n$ with $\lambda$ fixed, the first and second largest components exhibit complex chaotic behaviour. On the other

Date: 3 January 2009 (October 31, 2018).

2000 Mathematics Subject Classification. 05C80.

Key words and phrases. random graphs, percolation, phase transition, scaling window. 
hand, in the barely supercritical phase, just after the critical window, the dominant, or 'giant', component will have asserted itself.

In the present paper, our object of study is the 2-dimensional Hamming graph $H(2, n)$. The $n^{2}$ vertices of this graph can be represented as ordered pairs $(i, j), 1 \leq i, j \leq n$. Vertices $(i, j)$ and $\left(i^{\prime}, j^{\prime}\right)$ are adjacent if and only if either $i=i^{\prime}$ or $j=j^{\prime}$. Pictorially, $H(2, n)$ consists of an $n \times n$ lattice with each horizontal and vertical line being a complete graph. We write $\Omega=2(n-1)$ for the vertex degree in $H(2, n)$. We examine random subgraphs of $H(2, n)$ in independent percolation with edge probability $p$; that is, each edge is kept with probability $p$ and removed with probability $1-p$, independently of all other edges. We set $p_{c}=1 / \Omega$, which will act as our critical probability; a justification for this definition of critical probability lies in the recent results in [3, 4, 7]. We parametrise $p=(1+\varepsilon) / \Omega$ so that the average vertex degree is $1+\varepsilon$. Throughout the rest of the paper $C_{(1)}, C_{(2)}$ will refer to the largest and second largest components, respectively, of the Hamming graph $H(2, n)$. Also, we shall use the phrase 'with high probability' (whp) to mean 'with probability $1-o(1)$ as $n \rightarrow \infty$ '.

This work continues the exploration of van der Hofstad and Luczak [7]. It was shown therein that, when $n^{-2 / 3}(\log n)^{1 / 3} \ll \varepsilon \ll 1$, the largest component has size $2 \varepsilon n^{2}\left(1+o_{\mathrm{p}}(1)\right)$. The general sense of a mean-field percolation event in percolation on a graph with $V$ vertices is that there is a critical probability $p_{c}$, and that the barely supercritical phase occurs when $p=p_{c}(1+\varepsilon)$ and $V^{-1 / 3} \ll \varepsilon \ll 1$. This is the case in the Erdös-Rényi phase transition with $V=n$. For the $H(2, n)$ phase transition, $V=n^{2}$, and so the above results, up to a logarithmic term, fit the mean-field paradigm. Here we study the second largest component in percolation on $H(2, n)$ in the barely supercritical region. In this aspect we are also able to, again up to a logarithmic term, fit the mean-field paradigm. In the mean-field picture of random graphs, the structure remaining when the dominant component is removed for $p=p_{c}(1+\varepsilon)$ where $\varepsilon=\varepsilon(n) \rightarrow 0$ is like the largest connected component in the subcritical regime with $p=p_{c}(1-\varepsilon)$. It is well known that in this regime in $G(n, p)$, the second largest component is of order $\varepsilon^{-2} \log \left(n \varepsilon^{3}\right)$. The upper bound is the content of our main result. In our results for $H(2, n)$, we shall always work at $p=p_{c}+\varepsilon / \Omega=p_{c}+\varepsilon /(2(n-1))$. In [7, (1.10) and (1.11)], it is shown that there is little difference in working with $p=p_{c}+\varepsilon / \Omega$ or $p=(1+\varepsilon) / \Omega$, and we refer the reader there for more details.

Theorem 1.1 (The second component in the supercritical phase for $H(2, n)$ ). Consider the 2dimensional Hamming graph $H(2, n)$. Let $p=p_{c}+\frac{\varepsilon}{\Omega}$ and let $n^{-2 / 3}(\log n)^{1 / 3} \ll \varepsilon \ll 1$. Then, whp,

$$
\left|C_{(2)}\right| \leq 2^{8} \varepsilon^{-2} \log \left(n^{2} \varepsilon^{3}\right) .
$$

In particular, this result implies that the ratio of the sizes of the second and first largest components tends to zero in this regime, a salient feature of the barely supercritical phase. We feel that this feature should hold even without the logarithmic separation from criticality. That is, parametrise $2(n-1) p=1+\varepsilon$ and assume only $n^{-2 / 3} \ll \varepsilon \ll 1$. We conjecture, following [7], that the largest component will have size $2 \varepsilon n^{2}\left(1+o_{\mathrm{p}}(1)\right)$. We further conjecture that the second largest component will have size $\ll n^{2 / 3}$, which in particular is asymptotically smaller than the largest component. Let us note at this point that the logarithmic gap from the critical window (defined as in [3, 4, 5]) has recently been removed by Asaf Nachmias [11]; however, he does not establish a law of large numbers for the giant component, and he does not consider the second largest component. Further, we conjecture that, when $n^{-2 / 3} \ll \varepsilon \ll 1,\left|C_{(2)}\right|=\Theta\left(\varepsilon^{-2} \log \left(n^{2} \varepsilon^{3}\right)\right)$, i.e., the bound in Theorem 1.1 is sharp. Thus, in particular, we conjecture that the barely supercritical regime for $H(n, 2)$ has similar behaviour to that of $G(n, p)$. 


\section{PREliminaries}

In this section, we establish a lemma for a class of branching processes that will play a key role in our proofs.

We start with an inequality concerning deviations of binomial random variables below their mean. If $X \sim \operatorname{Bi}(k, p)$, then (see for instance [8])

$$
\mathbb{P}(X \leq k p-t) \leq e^{-\frac{t^{2}}{2\left(k p+\frac{t}{3}\right)}}
$$

We consider Galton-Watson processes where each individual's offspring is a random variable $Z$ such that $\mathbb{E}\left[Z^{2}\right]<\infty$. We always assume that our process begins with one individual. Sometimes we shall take $Z$ to have a binomial distribution $\operatorname{Bi}(N, p)$, with $p$ the Hamming graph edge probability, and $N$ a suitable positive integer. We will write $\mathbb{P}_{N, p}$ for the probability measure corresponding to this process. We will also need Galton-Watson processes that are 'inhomogeneous', in that the offspring size may vary depending on the parent's 'location' in the Galton-Watson tree.

A Galton-Watson process can be thought of as a 2-dimensional Markov chain $\left(Q_{t}, G_{t}\right)$, where $Q_{t}$ is the total progeny born until time $t$, and $G_{t}$ is the total number of 'active' population members, that is those that are yet to have offspring. To be precise, we think of a Galton-Watson process as an evolving tree that is explored one node at a time; then $Q_{t}$ is the total number of nodes in that tree at time $t$, and $G_{t}$ is the total number of unexplored nodes at time $t$. At each time $t$, if $G_{t}>0$, then we choose one active member of the population and decide the number of its offspring. In a homogeneous Galton-Watson process, all population members have the same offspring distribution, in our case binomial distribution $\operatorname{Bi}(N, p)$, where $N \in \mathbb{N}$ and $p \in[0,1]$ is the Hamming graph edge probability. Then, on the event $\left\{\omega: G_{t}(\omega) \geq 1\right\}$,

$$
Q_{t+1}=Q_{t}+Z_{t}, \quad G_{t+1}=G_{t}+Z_{t}-1,
$$

where the $Z_{t}$ are i.i.d. $\operatorname{Bi}(N, p)$. We always assume $Q_{0}=G_{0}=1$; we let $\varphi_{0}=\varnothing$ and

$$
\varphi_{t}=\sigma\left(Z_{s}: s \leq t\right), \quad t=1,2, \ldots,
$$

the $\sigma$-field generated by $Z_{s}(s=1,2, \ldots, t)$.

Note that, by the above,

$$
\begin{aligned}
& \mathbb{I}_{G_{t-1} \geq 1} Q_{t}=\mathbb{I}_{G_{t-1} \geq 1}\left(1+Z_{0}+\ldots+Z_{t-1}\right), \\
& \mathbb{I}_{G_{t-1} \geq 1} G_{t}=\mathbb{I}_{G_{t-1} \geq 1}\left(1+Z_{0}+\ldots+Z_{t-1}-t\right),
\end{aligned}
$$

where $\mathbb{I}_{\mathcal{A}}$ denotes the indicator of the event $\mathcal{A}$. Letting $T_{0}=\inf \left\{t: G_{t}=0\right\}$, we further see that

$$
Z_{0}+\ldots+Z_{T_{0}-1}=T_{0}-1 .
$$

For convenience, we shall instead assume that our Galton-Watson processes have a continuation in that the random variables $Z_{t}$ continue to be generated even after $G_{t}$ has hit 0 . With this assumption, we may simply write, for each $t$,

$$
Q_{t}=1+Z_{0}+\ldots+Z_{t-1}, \quad G_{t}=1+Z_{0}+\ldots+Z_{t-1}-t .
$$

We shall now generalise this setting to a branching process where $Z_{t}$ are not i.i.d., but where each $Z_{t}$ is Binomial $\operatorname{Bi}\left(N_{t}, p\right)$. Here, $p$ is the edge probability of the Hamming graph $H(2, n)$, and each $N_{t}$ is a random variable independent of $Z_{t}$ and such that, for each $t$, and conditionally on $\varphi_{t-1}$,

$$
N_{t} p \in[1+\varepsilon / 2,1+\varepsilon] \quad \text { with probability } 1 \text {. }
$$


Any such branching process will be called generically a narrow-banded (discrete) birth process, and in the lemma below we use $\mathbb{P}$ to denote the corresponding probability measure - with a slight abuse of notation, since in fact there is a whole class of processes satisfying property (2.8).

Lemma 2.1 (Large finite progeny for narrow-banded processes). Let $Q$ be the total population size of a narrow-banded birth process as defined above. Then, for $\alpha>0$ sufficiently large, there exists a constant $C$ such that

$$
\mathbb{P}\left(\alpha \varepsilon^{-2} \leq Q<\infty\right)<C \varepsilon e^{-\alpha / 2^{8}} .
$$

Proof. As described above, at each time $t$ we generate $Z_{t}$, a binomial $\operatorname{Bi}\left(N_{t}, p\right)$, where

$$
p=\frac{1+\varepsilon}{2(n-1)} \quad \text { and } \quad \mathbb{P}\left(N_{t} p \in[1+\varepsilon / 2,1+\varepsilon] \mid \varphi_{t-1}\right)=1 .
$$

As earlier in this section, let $G_{0}=1$, and let $G_{t}=1+Z_{0}+\ldots+Z_{t-1}-t$ for $t=1,2, \ldots$ In other words, we assume our narrow-banded Galton-Watson process to have a continuation; clearly, this does not in any way affect the correctness of the proof of the lemma.

Let

$$
\begin{aligned}
& \mathcal{E}=\left\{G_{\left\lfloor\alpha \varepsilon^{-2} / 2\right\rfloor}<\alpha \varepsilon^{-1} / 16 ; \quad G_{t}>0 \text { for all } t \leq \alpha \varepsilon^{-2} / 2\right\}, \\
& \tilde{\mathcal{E}}=\left\{G_{\left\lfloor\alpha \varepsilon^{-2} / 2\right\rfloor} \geq \alpha \varepsilon^{-1} / 16 ; \quad G_{t}>0 \text { for all } t \leq \alpha \varepsilon^{-2} / 2\right\} .
\end{aligned}
$$

Let $T_{0}$ be the first time $t$ such that $G_{t}=0$. Then $T_{0} \leq \alpha \varepsilon^{-2} / 2$, and $G_{T_{0}-1}=1$, so it follows that

$$
Q=Q_{T_{0}-1}=G_{T_{0}-1}+T_{0}-1 \leq \alpha \varepsilon^{-2} / 2<\alpha \varepsilon^{-2} \text {. }
$$

Therefore, we can upper bound

$$
\begin{aligned}
\mathbb{P}\left(\alpha \varepsilon^{-2} \leq Q<\infty\right) & =\mathbb{P}\left(\left\{\alpha \varepsilon^{-2} \leq Q<\infty\right\} \cap \mathcal{E}^{c}\right)+\mathbb{P}\left(\left\{\alpha \varepsilon^{-2} \leq Q<\infty\right\} \cap \mathcal{E}\right) \\
& \leq \mathbb{P}\left(\left\{\alpha \varepsilon^{-2} \leq Q<\infty\right\} \cap \tilde{\mathcal{E}}\right)+\mathbb{P}(\mathcal{E}) \\
& =\mathbb{P}\left(\alpha \varepsilon^{-2} \leq Q<\infty \mid \tilde{\mathcal{E}}\right) \mathbb{P}(\tilde{\mathcal{E}})+\mathbb{P}(\mathcal{E}),
\end{aligned}
$$

since, if $Q \geq \alpha \varepsilon^{-2}$, then $G_{t}>0$ for all $t \leq \alpha \varepsilon^{-2} / 2$. We shall start by bounding $\mathbb{P}\left(\alpha \varepsilon^{-2} \leq Q<\infty \mid\right.$ $\tilde{\mathcal{E}})$, and later bound $\mathbb{P}(\mathcal{E})$ and $\mathbb{P}(\tilde{\mathcal{E}})$.

On the event $\tilde{\mathcal{E}}$, for $t \geq \alpha \varepsilon^{-2} / 2$, we couple our narrow-banded birth process with the standard Galton-Watson process where the offspring distribution is binomial

$$
\operatorname{Bi}\left(\left\lfloor\frac{2(n-1)(1+\varepsilon / 2)}{1+\varepsilon}\right\rfloor, \frac{1+\varepsilon}{2(n-1)}\right),
$$

with mean less than or equal to $1+\varepsilon / 2$. Let $Q_{t}^{-}$and $G_{t}^{-}$respectively be the total progeny and total number of active members at time $t$ for this process; assume also that $Q_{\left\lfloor\alpha \varepsilon^{-2} / 2\right\rfloor}^{-}=\left\lceil\alpha \varepsilon^{-1} / 16\right\rceil$. Let $t_{0}=\left\lfloor\alpha \varepsilon^{-2} / 2\right\rfloor$, and for $t \geq t_{0}+1$ let us write

$$
Q_{t}^{-}=\left\lceil\alpha \varepsilon^{-1} / 16\right\rceil+Z_{t_{0}}^{-}+\ldots+Z_{t}^{-}, \quad G_{t}^{-}=\left\lceil\alpha \varepsilon^{-1} / 16\right\rceil+Z_{t_{0}}^{-}+\ldots+Z_{t}^{-}-\left(t-t_{0}\right),
$$

where the $Z_{t}^{-}$are i.i.d. $\operatorname{Bi}\left(\lfloor(2(n-1)(1+\varepsilon / 2) /(1+\varepsilon)\rfloor,(1+\varepsilon) / 2(n-1))\right.$. Let $\mathbb{P}^{-}$denote the probability measure corresponding to this process.

The coupling is between the corresponding tree exploration processes, step-by-step, as is standard (and as used, for instance, in [7, Section 4]), so that $Z_{t} \geq Z_{t}^{-}, Q_{t} \geq Q_{t}^{-}$and $G_{t} \geq G_{t}^{-}$for all $t \geq\left\lfloor\alpha \varepsilon^{-2} / 2\right\rfloor$. This implies that

$$
\mathbb{P}\left(\sup _{t} Q_{t}<\infty \mid \tilde{\mathcal{E}}\right) \leq \mathbb{P}^{-}\left(\sup Q_{t}^{-}<\infty\right) .
$$


Let $\mathbb{Q}_{k}$ denote the law of a branching process with offspring distribution in (2.13), and starting from $k$ individuals, and let $\tilde{Q}^{-}$be its total progeny. Then,

$$
\mathbb{P}^{-}\left(\sup Q_{t}^{-}<\infty\right)=\mathbb{Q}_{\left\lceil\alpha \varepsilon^{-1} / 16\right\rceil}\left(\tilde{Q}^{-}<\infty\right)=\mathbb{Q}_{1}\left(\tilde{Q}^{-}<\infty\right)^{\left\lceil\alpha \varepsilon^{-1} / 16\right\rceil},
$$

by the independence of the evolution of the initial individuals.

Further, [7, (3.28)] shows that

$$
\mathbb{Q}_{1}\left(\tilde{Q}^{-}<\infty\right)=1-\varepsilon+O\left(n^{-1}+\varepsilon^{2}\right),
$$

so that

$$
\mathbb{P}^{-}\left(\sup Q_{t}^{-}<\infty\right)=\left(1-\varepsilon+O\left(n^{-1}+\varepsilon^{2}\right)\right)^{\left\lceil\alpha \varepsilon^{-1} / 16\right\rceil} \leq e^{-\alpha / 16}\left(1+O\left(\alpha\left(\varepsilon+(\varepsilon n)^{-1}\right)\right)\right),
$$

for all $\varepsilon \in(0,1)$ and $\alpha>0$. We conclude from the above thet

$$
\mathbb{P}\left(\sup _{t} Q_{t}<\infty \mid \tilde{\mathcal{E}}\right) \leq e^{-\alpha / 16}(1+o(1))
$$

Next we shall show that $\mathbb{P}(\mathcal{E})$ and $\mathbb{P}(\tilde{\mathcal{E}})$ are quite small. For this, we also need an upper bounding Galton-Watson process $\left(Q_{t}^{+}, G_{t}^{+}\right)$, where the offspring distribution is binomial

$$
\operatorname{Bi}\left(2(n-1), \frac{1+\varepsilon}{2(n-1)}\right) \text {, }
$$

with mean $1+\varepsilon$. Let $Q_{t}^{+}$and $G_{t}^{+}$respectively be the total progeny and total number of active members at time $t$ for this process; assume also that the initial population size is $Q_{0}^{+}=1$. Let us write

$$
Q_{t}^{+}=1+Z_{0}^{+}+\ldots+Z_{t-1}^{+}, \quad G_{t}^{+}=1+Z_{0}^{+}+\ldots+Z_{t-1}^{+}-t,
$$

where the $Z_{t}^{+}$are i.i.d. $\operatorname{Bi}(2(n-1),(1+\varepsilon) / 2(n-1))$. We use $\mathbb{P}^{+}$to denote the corresponding probability measure.

Similarly, we use a lower bounding Galton-Watson process $\left(Q_{t}^{-}, G_{t}^{-}\right)$(but this time starting from time 0 , rather than from time $\left.t_{0}=\left\lfloor\alpha \varepsilon^{-2} / 2\right\rfloor\right)$, where the offspring distribution is binomial $\mathrm{Bi}(\lfloor 2(n-1)(1+\varepsilon / 2) /(1+\varepsilon)\rfloor,(1+\varepsilon) / 2(n-1))$, with mean at most $1+\varepsilon / 2$. Let $Q_{t}^{-}$and $G_{t}^{-}$be the total progeny and total number of active members at time $t$ for this process; assume also that the initial population size is $Q_{0}^{-}=1$. Let us write

$$
Q_{t}^{-}=1+Z_{0}^{-}+\ldots+Z_{t-1}^{-}, \quad G_{t}^{-}=1+Z_{0}^{-}+\ldots+Z_{t-1}^{-}-t,
$$

where the $Z_{t}^{-}$are i.i.d. $\operatorname{Bi}(\lfloor 2(n-1)(1+\varepsilon / 2) /(1+\varepsilon)\rfloor,(1+\varepsilon) / 2(n-1))$. Once again, we use $\mathbb{P}^{-}$ to denote the corresponding probability measure.

Now we couple $Z_{t}$ with $Z_{t}^{-}$and $Z_{t}^{+}$, so that, for all $t=0,1, \ldots$,

$$
Z_{t}^{-} \leq Z_{t} \leq Z_{t}^{+}
$$

A suitable coupling can be achieved thanks to standard results about stochastic domination between binomial random variables with different parameters. Explicitly, for $n$ large enough, we may generate independent binomial random variables $Z_{t}^{-}$such that

$$
Z_{t}^{-} \sim \operatorname{Bi}(\lfloor 2(n-1)(1+\varepsilon / 2) /(1+\varepsilon)\rfloor,(1+\varepsilon) / 2(n-1)),
$$

and independent binomial random variables $W_{t}$ such that

$$
W_{t} \sim \operatorname{Bi}(2(n-1)-\lfloor 2(n-1)(1+\varepsilon / 2) /(1+\varepsilon)\rfloor,(1+\varepsilon) / 2(n-1)) .
$$

We can then set $Z_{t}^{+}=Z_{t}^{-}+W_{t}$ for all $t$. We let $\mathbb{P}^{+,-}$denote the coupling measure. 
Let $\mathcal{A}$ be the event that $G_{t}^{+}>0$ for all $t \leq t_{0}$. Let $\mathcal{B}$ be the event that $G_{t_{0}}^{-}<\alpha \varepsilon^{-1} / 16$. Note that, under the coupling,

$$
\mathcal{E} \subseteq \mathcal{A} \cap \mathcal{B}
$$

However, now it is easily seen (using [7, Proposition 3.2]) that there exists $c_{0}=c_{0}(\alpha)$ such that $c_{0} \rightarrow 0$ as $\alpha \rightarrow \infty$ and

$$
\mathbb{P}_{p}^{+,-}(\mathcal{A})=\mathbb{P}_{p}\left(Q \geq t_{0}\right)=2 \varepsilon+O\left(\varepsilon^{2}+1 / \sqrt{t_{0}}\right) \leq\left(2+c_{0}(\alpha)\right) \varepsilon(1+o(1)) .
$$

This follows since $t_{0}=\left\lfloor\alpha \varepsilon^{-2} / 2\right\rfloor$. Also, using (2.1),

$$
\begin{aligned}
\mathbb{P}_{p}^{+,-}(\mathcal{B}) & \leq \mathbb{P}_{p}\left(\operatorname{Bi}\left(\lfloor 2(n-1)(1+\varepsilon / 2) /(1+\varepsilon)\rfloor\left\lfloor\alpha \varepsilon^{-2} / 2\right\rfloor,(1+\varepsilon) / 2(n-1)\right)<\alpha \varepsilon^{-2} / 2+\alpha \varepsilon^{-1} / 16\right) \\
& \leq \exp \left(-\alpha / 2^{8}\right),
\end{aligned}
$$

for all $\varepsilon$ satisfying $\varepsilon \gg n^{-2 / 3}(\log n)^{1 / 3}$, and all $\alpha$ and $n$ sufficiently large, since $\alpha \varepsilon^{-1} \gg 1$ and $\alpha \varepsilon^{-1} \gg \alpha \varepsilon^{-2} / n$.

Now, the event $\mathcal{A}$ is increasing, and the event $\mathcal{B}$ is decreasing, and both are events on the same probability space, corresponding to a family of independent random variables. It then follows from the FKG inequality that they are negatively correlated. Hence,

$$
\mathbb{P}_{p}(\mathcal{E}) \leq \mathbb{P}_{p}^{+,-}(\mathcal{A} \cap \mathcal{B}) \leq \mathbb{P}_{p}^{+,-}(\mathcal{A}) \mathbb{P}_{p}^{+,-}(\mathcal{B}) \leq\left(2+c_{0}(\alpha)\right) \varepsilon e^{-\alpha / 2^{8}}(1+o(1))
$$

Also,

$$
\mathbb{P}(\tilde{\mathcal{E}}) \leq \mathbb{P}_{p}^{+,-}(\mathcal{A})
$$

and hence

$$
\mathbb{P}_{p}\left(\alpha \varepsilon^{-2} \leq Q<\infty\right) \leq \mathbb{P}\left(\left\{\alpha \varepsilon^{-2} \leq Q<\infty\right\} \cap \tilde{\mathcal{E}}\right)+\mathbb{P}(\mathcal{E}) \leq 2\left(2+c_{0}(\alpha)\right) \varepsilon e^{-\alpha / 2^{8}}(1+o(1)) .
$$

\section{Proof OF MAIN RESUlT}

Recall that $\Omega=2(n-1)$. Let $Q(\mathbf{v})$ denote the component of vertex $\mathbf{v}$. Our first lemma is $[7$, Proposition 2.1].

Lemma 3.1 (Cluster tail equals the survival probability). Let $\varepsilon$ satisfy $\varepsilon^{3} n^{2} \gg \log n$. Let $p=$ $p_{c}+\varepsilon / \Omega$. Let $N \gg \varepsilon^{-2}$. Then, for any vertex $\mathbf{v}_{0}=\left(i_{0}, j_{0}\right)$,

$$
\mathbb{P}_{p}\left(\left|Q\left(\mathbf{v}_{0}\right)\right| \geq N\right)=2 \varepsilon(1+o(1)) .
$$

Our next lemma upper bounds the variance of $Z_{\geq N}$, the number of vertices in components at least $N$. This result is a special case of [7, Corollary 2.3].

Lemma 3.2 (Concentration of vertices in large clusters). Let $\varepsilon$ satisfy $\varepsilon^{3} n^{2} \gg \log n$. Let $p=$ $p_{c}+\varepsilon / \Omega$. Let $N \gg \varepsilon^{-2}$. Then, for every $\delta>0$,

$$
\mathbb{P}_{p}\left(\left|Z_{\geq N}-\mathbb{E}_{p}\left[Z_{\geq N}\right]\right| \geq \delta \varepsilon n^{2}\right)=o(1) .
$$

We now show that, whp, there are no components of 'medium' size; that is, if $\alpha=\alpha(n) \rightarrow \infty$ as $n \rightarrow \infty$, then any component of size at least $\alpha \varepsilon^{-2}$, whp will in fact be of size at least $\varepsilon n^{2} / 5$. This is the content of our next lemma: 
Lemma 3.3 (No middle ground). Let $\varepsilon$ satisfy $\varepsilon^{3} n^{2} \gg \log n$. Let $p=p_{c}+\varepsilon / \Omega$. Let $\alpha=\alpha(n) \rightarrow \infty$ as $n \rightarrow \infty$. Then there exists a constant $C$ such that, for $n$ large enough,

$$
\mathbb{P}_{p}\left(\alpha \varepsilon^{-2} \leq\left|Q\left(\mathbf{v}_{\mathbf{0}}\right)\right|<\frac{\varepsilon n^{2}}{5}\right) \leq C\left(\varepsilon e^{-\alpha / 2^{8}}+n^{-6}\right)
$$

Hence, the probability that there is some vertex $\mathbf{v}_{\mathbf{0}}$ such that its component $Q\left(\mathbf{v}_{\mathbf{0}}\right)$ satisfies

$$
2^{8} \varepsilon^{-2} \log \left(n^{2} \varepsilon^{3}\right) \leq\left|Q\left(\mathbf{v}_{\mathbf{0}}\right)\right|<\varepsilon n^{2} / 5
$$

is o(1) as $n \rightarrow \infty$.

Before giving the proof of Lemma 3.3, let us state two more results from [7], which compare the size of the cluster of a vertex to the total progeny of suitable Galton-Watson processes.

The first of these is essentially [7, Lemma 4.1], proved by standard methods, and gives an upper bound. Let $\mathbb{P}_{\Omega, p}$ be the probability measure corresponding to a standard Galton-Watson process where the family size is a binomial with parameters $\Omega$ and $p$, and the initial population size is 1 .

Lemma 3.4 (Stochastic domination of cluster size by branching process progeny size). For every $\ell \in \mathbb{N}$,

$$
\mathbb{P}_{p}\left(\left|Q\left(\mathbf{v}_{0}\right)\right| \geq \ell\right) \leq \mathbb{P}_{\Omega, p}(Q \geq \ell)
$$

The second one is a slight extension of [7, Lemma 4.3], and establishes a lower bound. Let $\Omega^{\prime}=\Omega-\frac{5}{2} \max \left\{\ell n^{-1}, C \log n\right\}$ and note that $\Omega^{\prime} \geq 2(n-1)-\frac{1}{2} \varepsilon n$ for $n$ sufficiently large. It turns out that the cluster size can be stochastically bounded from below using a Galton-Watson process where the family size is a binomial with parameters $\Omega^{\prime}$ and $p$, and the initial population size is 1 . For $n$ sufficiently large, this process is supercritical, with mean population size at least $1+\varepsilon / 2$, since $\Omega^{\prime} \geq 2(n-1)-\frac{1}{2} \varepsilon n$.

Lemma 3.5 (Stochastic domination of cluster size over branching process progeny size). There is a constant $C>0$ such that the following holds. For every $\ell \leq \varepsilon n^{2} / 5$,

$$
\mathbb{P}_{p}\left(\left|Q\left(\mathbf{v}_{0}\right)\right| \geq \ell\right) \geq \mathbb{P}_{\Omega^{\prime}, p}(Q \geq \ell)+O\left(n^{-6}\right),
$$

where $\Omega^{\prime}=\Omega-\frac{5}{2} \max \left\{\ell n^{-1}, C \log n\right\} \geq 2(n-1)-\frac{1}{2} \varepsilon n$.

Lemma 3.5 can be proved in exactly the same way as [7, Lemma 4.3], using an extension of [7. Proposition 4.4] concerning the number of elements per line in large clusters from $\eta \ll \varepsilon$ to $\eta \leq \varepsilon / 5$ (which is exactly the same, again, since the proof of [7, Proposition 4.4] does not in any way rely on $\eta$ being of a smaller order than $\varepsilon$ ).

In fact, Lemmas 3.4 and 3.5 are not sufficient for our purposes, and we refine them in the following. Let $Q_{t}\left(\mathbf{v}_{\mathbf{0}}\right), G_{t}\left(\mathbf{v}_{\mathbf{0}}\right)$ denote the total number of vertices and the number of unexplored vertices at time $t$ in the exploration of the cluster of vertex $\mathbf{v}_{\mathbf{0}}$. Also, $Q_{t}$ and $G_{t}$, respectively, will denote the total number of nodes and the number of unexplored nodes at time $t$ in the GaltonWatson tree when the offspring is binomial $\operatorname{Bi}(\Omega, p)$; and let $Q_{t}^{\prime}$ and $G_{t}^{\prime}$, respectively, denote the total number of nodes and the number of unexplored nodes at time $t$ in the Galton-Watson tree when the offspring is binomial $\operatorname{Bi}\left(\Omega^{\prime}, p\right)$. Let $\mathcal{E}_{t}$ be the event that, for every $i$, no more than $m$ vertices $(i, x)$ and no more than $m$ vertices $(x, i)$ have been included in the cluster of a vertex $\mathbf{v}_{\mathbf{0}}$ up to time $t$ during its exploration process. Also, let $\mathcal{E}_{t}^{\prime}$ be the event that $Q_{t}^{\prime} \leq Q_{t}\left(\mathbf{v}_{\mathbf{0}}\right) \leq Q_{t}$ and $G_{t}^{\prime} \leq G_{t}\left(\mathbf{v}_{0}\right) \leq G_{t}$.

Lemma 3.6 (Sandwiching the cluster exploration). Let $\ell=\varepsilon n^{2} / 5$, and let $m=5 \ell n^{-1} / 2$. Then, if $n$ is large enough, on the event $\mathcal{E}_{t} \cap \mathcal{E}_{t}^{\prime}$, there exists a coupling $\mathbb{P}_{\Omega, \Omega^{\prime}, p}$ of the cluster exploration 
process and the upper and lower bounding Galton-Watson processes $\mathbb{P}_{\Omega, p}, \mathbb{P}_{\Omega^{\prime}, p}$ in such a way that, $\mathbb{P}_{\Omega, \Omega^{\prime}, p^{-}}$almost surely, $Q_{t+1}^{\prime} \leq Q_{t+1}\left(\mathbf{v}_{\mathbf{0}}\right) \leq Q_{t+1}$ and $G_{t+1}^{\prime} \leq G_{t+1}\left(\mathbf{v}_{\mathbf{0}}\right) \leq G_{t+1}$.

It is easy to prove Lemma 3.6 using standard component exploration and coupling methods, in a similar way to [7, Lemmas 4.1 and 4.3], and so we omit the details. We are now ready to prove that there is indeed no middle ground:

Proof of Lemma 3.3. Lemma 3.6 implies that, on the event $\mathcal{E}_{t} \cap \mathcal{E}_{t}^{\prime}$, the $(t+1)^{\text {th }}$ step of the exploration process of the cluster of vertex $\mathbf{v}_{\mathbf{0}}$ can be coupled with the $(t+1)^{\text {th }}$ step of a narrowbanded process in Lemma 2.1. Since $\left[2(n-1)-\frac{1}{2} \varepsilon n\right] p \geq 1+\varepsilon / 2$ for $n$ large enough, the family size of the narrow-banded branching process in question (i.e., the component exploration process) falls into the interval $[1+\varepsilon / 2,1+\varepsilon]$, as required. Now observe that $Q\left(\mathbf{v}_{\mathbf{0}}\right) \geq \ell$ if and only if $Q_{\ell}\left(\mathbf{v}_{\mathbf{0}}\right) \geq \ell$. We use this fact, first with $\ell=2^{8} \varepsilon^{-2} \log \left(n^{2} \varepsilon^{-3}\right)$, and then with $\ell=\varepsilon^{2} n / 5$. Then, the first claim follows directly from Lemma 2.1, also noting that $\mathbb{P}_{p}\left(\mathcal{E}_{t}^{c}\right)=O\left(n^{-6}\right)$ for all $t \leq \varepsilon n^{2} / 5$, see [7, Proposition 4.4 and its proof].

As for the second claim, note that for every $x, y$, the number of components of size in between $x$ and $y$, where $0 \leq x \leq y$, is given by

$$
N_{x, y}=\sum_{\mathbf{v}} \frac{1}{|Q(\mathbf{v})|} \mathbb{I}_{x \leq|Q(\mathbf{v})|<y} .
$$

Let $x=2^{8} \varepsilon^{-2} \log \left(n^{2} \varepsilon^{3}\right)$ and $y=\varepsilon n^{2} / 5$. Then, for any vertex $\mathbf{v}$,

$$
\begin{aligned}
\mathbb{E}\left(\frac{1}{|Q(\mathbf{v})|} \mathbb{I}_{x \leq|Q(\mathbf{v})|<y}\right) & \leq \frac{2^{-8} \varepsilon^{2}}{\log \left(n^{2} \varepsilon^{3}\right)} \mathbb{P}_{p}\left(2^{8} \varepsilon^{-2} \log \left(n^{2} \varepsilon^{3}\right) \leq|Q(\mathbf{v})|<\varepsilon n^{2} / 5\right) \\
& \leq \frac{C 2^{-8} \varepsilon^{2}}{\log \left(n^{2} \varepsilon^{3}\right)}\left(\varepsilon e^{-\log \left(n^{2} \varepsilon^{3}\right)}+n^{-6}\right) \leq \frac{C}{n^{2} \log \left(n^{2} \varepsilon^{3}\right)}
\end{aligned}
$$

where, for the second inequality, we have used (3.3). Summing over all vertices $\mathbf{v}$, we see that $\mathbb{E}\left[N_{x, y}\right]=o(1)$, and hence $\mathbb{P}_{p}\left(N_{x, y} \geq 1\right)=o(1)$, as required.

We now complete the proof of Theorem 1.1.

Proof of Theorem 1.1. By Lemmas 3.1] 3.2, $Z_{\geq 2^{8} \varepsilon^{-2} \log \left(n^{2} \varepsilon^{3}\right)}$, the number of vertices in components of size at least $2^{8} \varepsilon^{-2} \log \left(n^{2} \varepsilon^{3}\right)$, is concentrated around $2 \varepsilon n^{2}$. In other words, the number of vertices in connected components of size at least $2^{8} \varepsilon^{-2} \log \left(n^{2} \varepsilon^{3}\right)$ is close to $2 \varepsilon n^{2}$ whp. Now, from [7], we know that, whp, there is a giant component of size $2 \varepsilon n^{2}\left(1+o_{\mathrm{p}}(1)\right)$. This implies that, whp, there is no other cluster of size at least $\varepsilon n^{2} / 5$. Further, by (3.4) in Lemma 3.3, whp, there are no components of size at least $2^{8} \varepsilon^{-2} \log \left(n^{2} \varepsilon^{3}\right)$ and less than $\varepsilon n^{2} / 5$. Hence, whp, the second largest component must be at most $2^{8} \varepsilon^{-2} \log \left(n^{2} \varepsilon^{3}\right)$, as claimed.

Acknowledgement. This work was started during a visit by MJL to NYU, and continued during a visit by MJL and JS to Georgia Tech, and during a visit by MJL to Eurandom. The hospitality of the three institutions is gratefully acknowledged. Also, the work of RvdH was supported in part by Netherlands Organisation for Scientific Research (NWO), and the work of MJL by the Nuffield Foundation.

\section{REFERENCES}

[1] B. Bollobás. The evolution of random graphs. Trans. Amer. Math. Soc., 286 (1984), 257-274.

[2] B. Bollobás. Random graphs, volume $\mathbf{7 3}$ of Cambridge Studies in Advanced Mathematics. Cambridge University Press, Cambridge, second edition, (2001). 
[3] C. Borgs, J.T. Chayes, R. van der Hofstad, G. Slade and J. Spencer, Random subgraphs of finite graphs: I. The scaling window under the triangle condition. Random Struct. Alg. 27 (2005) 137-184.

[4] C. Borgs, J.T. Chayes, R. van der Hofstad, G. Slade and J. Spencer, Random subgraphs of finite graphs: II.The lace expansion and the triangle condition. Ann. Probab. 33 (2005) 1886-1944.

[5] C. Borgs, J.T. Chayes, R. van der Hofstad, G. Slade and J. Spencer, Random subgraphs of finite graphs: III. The phase transition on the $n$-cube. Combinatorica, 26(4) (2006) 395-410.

[6] P. Erdös and A. Rényi, On the Evolution of the Random Graph, Magyar Tud. Akad. Mat. Kutató Int. Kozl. 5 (1960) $17-61$.

[7] R. van der Hofstad and M. J. Luczak. Random subgraphs of the 2D Hamming graph: the supercritical phase. Preprint 2007, to appear in Probab. Theor. Related Fields.

[8] S. Janson, On concentration of probability, Contemporary Combinatorics, ed. B. Bollobás, Bolyai Soc. Math. Stud. 10 (2002), János Bolyai Mathematical Society, Budapest, 289-301.

[9] S. Janson, T. Łuczak \& A. Ruciński, Random Graphs, Wiley, New York, 2000.

[10] T. Euczak. Component behavior near the critical point of the random graph process. Random Structures Algorithms, 1(3) (1990), 287-310.

[11] A. Nachmias, Mean-field conditions for percolation on finite graphs, Preprint 2007.

Department of Mathematics and Computer Science, Eindhoven University of Technology, 5600

MB Eindhoven, The Netherlands.

E-mail address: rhofstad@win.tue.nl

URL: http://www.win.tue.nl/ rhof stad

Department of Mathematics, London School of Economics, Houghton Street, London WC2A 2AE, United Kingdom

E-mail address: m.j.luczak@lse.ac.uk

$U R L:$ http://www.lse.ac.uk/people/m.j.luczak@lse.ac.uk/

Computer Science and Mathematics Departments, New York University, Mercer Street, New York City, United States of America.

E-mail address: spencer@courant.nyu.edu

$U R L:$ http://cs.nyu.edu/cs/faculty/spencer/ 